\title{
The modulatory effect of oscillatory reinstatement during slow- wave sleep on declarative memory consolidation
}

Rebecca Crowley ${ }^{1,2}$, Amir-Homayoun Javadi ${ }^{1,3,4}{ }^{*}$

${ }^{1}$ School of Psychology, University of Kent, Canterbury, UK

${ }^{2}$ Department of Psychology, Royal Holloway, University of London, London, UK

${ }^{3}$ Institute of Behavioural Neuroscience, University College London, London, UK

${ }^{4}$ School of Rehabilitation, Tehran University of Medical Sciences, Tehran, Iran

\section{*Corresponding author:}

$\begin{array}{ll} & \text { Amir-Homayoun Javadi } \\ \text { Address: } & \text { School of Psychology, } \\ & \text { Keynes College, } \\ & \text { University of Kent, } \\ & \text { CT2 7NP, Canterbury, } \\ & \text { Kent, UK } \\ & +441227827770 \\ \text { Phone: } & \text { a.h.javadi@ gmail.com } \\ \text { E-Mail: } & \end{array}$

Keywords: context-dependent memory, dorsolateral prefrontal cortex (DLPFC), engram reactivation, targeted memory reactivation (TMR), transcranial alternating current stimulation (tACS) 


\begin{abstract}
Consistent with the context-dependent memory literature, previous research suggests that when the same frequency of neural oscillations is reinstated between memory encoding and retrieval, engram reactivation is facilitated, and thus declarative memory recall is enhanced. Importantly, engram reactivation is also fundamental for the redistribution process that underlies sleep-dependent memory consolidation. Therefore, the current study investigated whether reinstating frequency-specific oscillatory activity between encoding and sleep would facilitate the engram reactivation implicated in sleep-dependent memory consolidation, and thus enhance post-sleep declarative memory performance. Transcranial alternating current stimulation (tACS) was administered to the left dorsolateral prefrontal cortex (DLPFC) of human participants during a declarative memory task. Participants received $60 \mathrm{~Hz}$ of stimulation during encoding, and $60 \mathrm{~Hz}, 90 \mathrm{~Hz}$, or sham stimulation during post-learning slow-wave sleep (SWS) or rapid eye-movement (REM) sleep. In immediate and delayed free recall sessions, declarative memory performance was significantly enhanced if participants had received the same frequency of stimulation during encoding and SWS compared to any other stimulation condition. This finding supports a novel theoretical proposal, which assumes that an intrinsic neurobiological mechanism for coordinating frequency-specific oscillatory activity, during SWS, underlies sleep-dependent declarative memory consolidation.
\end{abstract}


Memory enhancement refers to a phenomenon whereby an individual's memory for learned stimuli becomes greater compared to other learned stimuli (Kensinger \& Corkin, 2003). Since memory is a fundamental cognitive function that underlies a variety of tasks spanning the recall of survival-related information to the development of language (Gathercole \& Baddeley, 2014; Nairne, Thompson, \& Pandeirada, 2007), research has attempted to identify the neurobiological mechanisms that cause memory enhancement. On the one hand, reinstating implicit neural contexts between encoding and retrieval leads to memory enhancement by facilitating the retrieval process (Jafarpour, Fuentemilla, Horner, Penny, \& Duzel, 2014; Javadi, Glen, Halkiopoulos, Schulz, \& Spiers, 2017; Sederberg et al., 2007; Staresina et al., 2016; Wimber, Maßß, Staudigl, Richardson-Klavehn, \& Hanslmayr, 2012). On the other hand, memory enhancement is also achieved by facilitating sleep-dependent memory consolidation through the reinstatement of explicit environmental contexts between encoding and sleep (Antony, Gobel, O’Hare, Reber, \& Paller, 2012; Rasch, Büchel, Gais, \& Born, 2007; Smith \& Weeden, 1990; for reviews, see Oudiette \& Paller, 2013; Rasch \& Born, 2013). The current study combined these previous lines of research to determine whether implicit neural contexts also have a role in sleep-dependent memory consolidation, such that memories are enhanced following the reinstatement of frequency-specific neural oscillations between encoding and sleep.

According to the encoding specificity principle of memory, memory performance is dependent on encoding and retrieval conditions to the extent that recall is optimum when conditions that were present during stimulus encoding are also present during retrieval (Tulving \& Thomson, 1973). This principle underlies the concept of context-dependent memory whereby the reinstatement of contextual conditions between encoding and retrieval is thought to facilitate recall. Support for context-dependent memory comes from studies demonstrating that memory performance is greater when the environmental contexts that were present during encoding and retrieval were congruent rather than incongruent (Smith \& Vela, 2001). For example, in a seminal study, deep-sea divers learned and recalled a list of words in two environments: on land and underwater (Godden \& Baddeley, 1975). The results demonstrated that memory recall for words learned underwater was significantly greater if those words were also recalled underwater compared to on land, and similarly, words learned on land were better recalled on land compared to underwater. Hence, in line with contextdependent memory, memory performance was facilitated by congruency between explicit environmental contexts at encoding and retrieval. 
More recently, context-dependent memory has been reconceptualised within neurobiological memory models, which posit that memory performance is dependent on congruency between implicit neural contexts at encoding and retrieval (Marr, 1971; McClelland, McNaughton, \& O'Reilly, 1995; Norman \& O'Reilly, 2003; Teyler \& Rudy, 2007; Watrous \& Ekstrom, 2014; Watrous, Fell, Ekstrom, \& Axmacher, 2015; Watrous, Miller, Qasim, Fried, \& Jacobs, 2018). Specifically, the spectro-contextual encoding and retrieval theory of episodic memory (SCERT) predicts that the same frequency of neural oscillations (rhythmic fluctuations in cortical excitability; Fries, 2005) must occur between encoding and retrieval in order for memory recall to be optimum (Canavier, 2015; Hanslmayr \& Staudigl, 2014; Siegel, Donner, \& Engel, 2012; Sutterer, Foster, Serences, Vogel, \& Awh, 2018; Watrous et al., 2015; Watrous et al., 2018; Watrous \& Ekstrom, 2014). The reason being that frequency-specific oscillatory activity is assumed to modulate neural activity through the coordination of phase synchronisation and cross-frequency coupling.

Phase synchronisation (PS) and cross-frequency coupling (CFC) are neural mechanisms which relate to the oscillatory phases of neuronal networks. Specifically, PS refers to a phenomenon whereby the neural activity in one neuronal network peaks repeatedly at the same time in the oscillatory phase of another neuronal network (Fell \& Axmacher, 2011). Whereas, CFC occurs when the phase of lower-frequency neural oscillations modulates the amplitude of higher-frequency oscillations (Bragin et al., 1995; Canolty \& Knight, 2010). Importantly, ample evidence implicates PS and CFC as having functional roles in spike-timing dependent plasticity within neuronal networks as well as neural communication between neuronal networks (for reviews, see Canolty \& Knight, 2010; Fell \& Axmacher, 2011; Fries, 2005; Jutras \& Buffalo, 2010; Womelsdorf et al., 2007). Consequently, PS and CFC are thought to contribute to the formation and reactivation of neuronal groups that represent encoded events ('engrams'). Crucially, since frequencyspecificity is a notable feature of PS and CFC, SCERT predicts that if the frequency-specific oscillatory activity that occurred during memory encoding is reinstated during memory retrieval, engram reactivation will be facilitated, and thus memory recall will be enhanced.

The first causal evidence in support of SCERT's assumption that memory recall is facilitated by the reinstatement of neural oscillations was demonstrated using transcranial alternating current stimulation (tACS) to experimentally induce implicit neural contexts during encoding and retrieval (Javadi et al., 2017). Notably, tACS is a non-invasive electrical brain stimulation technique which has the capacity for neuronal entrainment whereby neural 
oscillations synchronise to the frequency of stimulation (Antal \& Paulus, 2013; Helfrich et al., 2014; Strüber, Rach, Trautmann-Lengsfeld, Engel, \& Hermann, 2014). In Javadi et al.'s (2017) study, participants performed a word recognition task, and tACS was administered to the left dorsolateral prefrontal cortex (DLPFC) at either the same or different gamma frequency during encoding and retrieval. The results found that compared to a sham stimulation condition, memory accuracy was significantly greater when participants received the same frequency of stimulation during encoding and retrieval $(60 \mathrm{~Hz} \& 60 \mathrm{~Hz}$ or $90 \mathrm{~Hz} \&$ $90 \mathrm{~Hz}$ ). However, no memory enhancement occurred between sham and active stimulation conditions if participants received different frequencies of stimulation during encoding and retrieval $(60 \mathrm{~Hz} \& 90 \mathrm{~Hz}$ or $90 \mathrm{~Hz} \& 60 \mathrm{~Hz})$. Henceforth, in line with SCERT, these findings coined 'oscillatory reinstatement' - indicate that reinstating the same frequency of neural oscillations between encoding and retrieval leads to the enhancement of memories by facilitating memory recall.

Whilst previous research suggests that engram reactivation during retrieval is fundamental for successful memory recall, a related line of research has also implicated a role for engram reactivation during sleep in memory consolidation. Specifically, the active system consolidation hypothesis assumes that memories are consolidated during sleep when recently encoded, fragile memory traces are redistributed from the hippocampus to the neocortex for storage as more persistent engrams (Born \& Wilhelm, 2012; Marshall \& Born, 2007; McClelland et al., 1995; O’Reilly \& Rudy, 2001; Walker \& Stickgold, 2006). Importantly, this redistribution process requires repeated reactivation of the neuronal groups during sleep that were active during encoding (Born \& Wilhelm, 2012; Diekelmann \& Born, 2010; Marshall \& Born, 2007; O’Neill, Pleydell-Bouverie, Dupret, \& Csicsvari, 2010; Rasch \& Born, 2013). Accordingly, Peigneux et al. (2004) demonstrated that hippocampal and parahippocampal activity, which occurred during spatial learning in a virtual navigation task, was subsequently reactivated during slow-wave sleep (SWS), and the amount of this reactivation correlated positively with the extent of improvement in post-sleep navigation performance.

Consequently, the memory enhancement literature has investigated whether techniques can be developed which experimentally induce engram reactivation during sleep in order to facilitate sleep-dependent memory consolidation, and thus enhance post-sleep memory performance. One such technique is targeted memory reactivation (TMR) whereby contextual cues are associated with stimuli during encoding, and these cues are subsequently 
re-presented during sleep in an attempt to reactivate specific engrams (Antony et al., 2012; Rasch et al., 2007; Smith \& Weeden, 1990; for reviews, see Oudiette \& Paller, 2013; Rasch \& Born, 2013; see also Ladenbauer et al., 2016; Ladenbauer et al., 2017; Paßmann et al., 2016). Using a TMR paradigm, Rasch et al. (2007) asked participants to perform a visuospatial card-pair (declarative) task and a finger-tapping (procedural) task in the presence of an olfactory stimulus, and the same olfactory stimulus or an odourless vehicle was represented during SWS, rapid eye-movement (REM) sleep, or wakefulness. The results found that not only was hippocampal activity greater when the olfactory stimulus was re-presented during SWS compared to wakefulness, but also post-sleep performance on the card-pair task was greater when the olfactory stimulus was re-presented during SWS compared to REM sleep, wakefulness, or when the odourless vehicle was presented. Moreover, no such finding occurred for the finger-tapping task or if the olfactory stimulus was not presented during encoding. Therefore, Rasch et al. (2007) concluded that re-presenting the olfactory stimulus during SWS facilitated participants' post-sleep declarative memory performance because hippocampal reactivation was elicited, which lead to increased consolidation of hippocampus-dependent (declarative) memories but not hippocampus-independent (procedural) memories. Henceforth, there is evidence demonstrating that memory enhancement is caused by the reinstatement of contextual conditions between encoding and sleep as well as between encoding and retrieval.

In line with the active system consolidation hypothesis, Rasch et al.'s (2007) findings suggest that increased engram reactivation during sleep was the mechanism which caused a memory enhancement following the reinstatement of explicit environmental contexts between encoding and sleep. As previously mentioned, SCERT assumes that engram reactivation during retrieval is facilitated by oscillatory reinstatement between encoding and retrieval, because frequency-specific oscillatory activity coordinates PS and CFC. Therefore, an interesting question that remains to be determined is whether oscillatory reinstatement also facilitates the engram reactivation that underlies sleep-dependent memory consolidation. Consequently, the current study investigated whether memory enhancement is induced following the reinstatement of frequency-specific oscillatory activity between encoding and sleep. Hence, this study aims to further SCERT's assumptions regarding the role of neural oscillations in encoding and retrieval by determining whether neural oscillations also have a role in sleep-dependent memory consolidation. Additionally, this study aims to introduce a 
brain stimulation protocol that could be used to enhance declarative memory performance for therapeutic purposes.

The current study used a similar procedure to Javadi et al. (2017) such that participants performed a declarative memory task involving word learning, and they received two sessions of tACS in which the same or different gamma frequency was administered to the left DLPFC. However, since the current study attempted to further Javadi et al.'s (2017) findings by investigating whether oscillatory reinstatement facilitates sleep-dependent memory consolidation, a novel stimulation protocol was used whereby tACS was administered during encoding and different stages of a 90-minute retention interval containing sleep. Moreover, the current study assessed post-sleep declarative memory performance using a free recall paradigm rather than cued recall or recognition paradigms for two reasons. Firstly, young adults often produce near-ceiling performance in post-sleep cued recall tasks, and therefore, statistical analyses have been limited in previous sleep studies (Ellenbogen, Hulbert, Stickgold, Dinges, \& Thompson-Schill, 2006; Rauchs et al., 2008; Scullin, 2013). Secondly, whereas post-learning sleep and TMR have been shown to have limited or no beneficial effects on subsequent recognition performance, free recall tasks tend to elicit pronounced memory enhancements following sleep and TMR (Ashton, Cairney, \& Gaskell, 2018; Diekelmann, Born, \& Rasch, 2016; Rauchs et al., 2004; Tamminen, Ralph, \& Lewis, 2017; Wang et al., 2017). Additionally, post-sleep declarative memory performance was assessed immediately after the 90-minute retention interval and 24 hours later, because further overnight processing has been found to attenuate sleep intervention-related memory benefits (Shimizu et al., 2018). Therefore, the current study aims to determine whether oscillatory reinstatement has a long-term modulatory effect on sleep-dependent declarative memory consolidation, such that differences in declarative memory performance between stimulation conditions are maintained following an additional night of sleep.

Importantly, stimulation during sleep was either administered during SWS or REM sleep. The reason being that ample evidence implicates SWS as having a fundamental role in declarative memory consolidation whereas REM sleep is implicated in the consolidation of procedural and emotional memories (Groch, Wilhelm, Diekelmann, \& Born, 2013; Pilhal \& Born, 1997, 1999; Wagner, Hallschmid, Verleger, \& Born, 2003; for reviews, see Ackermann \& Rasch, 2014; Gais \& Born, 2004; Rasch \& Born, 2013). Further, recent studies have demonstrated differential effects of TMR on declarative and non-declarative memory consolidation depending on whether the contextual cues were re-presented during SWS or 
REM sleep (Cairney, Durrant, Hulleman, \& Lewis, 2014; Rasch et al., 2007; Smith \& Weeden, 1990). These findings are all in line with the dual-process hypothesis of sleepdependent memory consolidation, which assumes that SWS and REM sleep contribute to declarative and non-declarative memory consolidation respectively (Maquet, 2001; Peigneux, Laureys, Delbeuck, \& Maquet, 2001). Henceforth, the current study aims to further investigate the relative contributions of SWS and REM sleep to declarative memory consolidation, by determining whether the potential effect of oscillatory reinstatement on sleep-dependent declarative memory consolidation is dependent on the stage of sleep in which reinstatement occurs.

The main prediction of the current study emanates from two lines of research regarding the facilitatory effect of oscillatory reinstatement on engram reactivation and the importance of engram reactivation for sleep-dependent memory consolidation. Specifically, it is hypothesised that sleep-dependent declarative memory consolidation, and thus post-sleep declarative memory performance, will be enhanced when the same frequency of neural oscillations is reinstated between encoding and sleep. Hence, participants who are administered with congruent stimulation during encoding and sleep should recall significantly more words than participants who are administered with incongruent stimulation or sham stimulation during encoding and sleep. Additionally, based on previous findings which suggest that SWS, rather than REM sleep, is responsible for declarative memory consolidation, it is hypothesised that oscillatory reinstatement will only enhance sleepdependent declarative memory consolidation if the reinstatement occurs during SWS as opposed to REM sleep. Hence, participants who receive congruent stimulation during encoding and SWS should recall significantly more words than participants who receive congruent stimulation during encoding and REM sleep. Therefore, these two hypotheses respectively investigate whether the effect of inducing neural oscillations on post-sleep declarative memory performance is frequency-specific and sleep stage-specific. Finally, TMR has been shown to modulate the contributions of various sleep stages, including sleep stages in which TMR was not administered, to memory consolidation (Cairney et al., 2014; Cousins, El-Deredy, Parkes, Hennies, \& Lewis, 2016; Tamminen et al., 2017). Consequently, the current study will also investigate relationships between the durations of specific sleep stages and post-sleep declarative memory performance. 


\section{Methods}

\section{Participants}

A total of 112 participants took part in this study for course credits or the chance to win an Amazon voucher. Data for 30 participants had to be excluded: 13 due to low performance in at least one of the test sessions (performance lower than the mean minus two SD of correct memory recall within their group), two due to a technical fault, 15 due to either waking during stimulation or a short total sleep duration (less than 60 minutes).

Therefore, data for 82 participants (mean age [SD] = 19.98 [2.16], age range 18-30, 62 females) is reported. All participants were naive to the aim of the study, spoke fluent English, and had normal or corrected-to-normal vision. Participants were screened to exclude those with a history of neurological or psychiatric disorder, and no participant was taking centrally acting medication. All participants gave their written informed consent, and the study was given ethical approval by the Psychology Research Ethical Committee, University of Kent.

Participants were randomly assigned to one of four conditions relating to the stimulation frequency and sleep stage in which tACS was to be administered: congruentSWS, incongruent-SWS, congruent-REM, and sham. Table 1 shows a breakdown of the stimulation conditions.

Table 1. Breakdown of stimulation conditions.

\begin{tabular}{lllll}
\hline Condition & n [\% female] & $\begin{array}{l}\text { Stim. freq. } \\
\text { during encoding }\end{array}$ & $\begin{array}{l}\text { Stim. freq. } \\
\text { during sleep }\end{array}$ & $\begin{array}{l}\text { Stim. stage } \\
\text { during sleep }\end{array}$ \\
\hline Congruent-SWS & $20[80.00]$ & $60 \mathrm{~Hz}$ & $60 \mathrm{~Hz}$ & SWS \\
Incongruent-SWS & $17[82.35]$ & $60 \mathrm{~Hz}$ & $90 \mathrm{~Hz}$ & SWS \\
Congruent-REM & $18[77.78]$ & $60 \mathrm{~Hz}$ & $60 \mathrm{~Hz}$ & REM \\
Sham & $27[66.67]$ & $60 \mathrm{~Hz}$ & None & None \\
\hline
\end{tabular}

notes: Stim. $=$ stimulation, freq. $=$ frequency, SWS $=$ slow-wave sleep, $\mathrm{REM}=$ rapid eye movement sleep. 


\section{Stimuli}

A set of 80 nouns and their corresponding images were created. All words were concrete and neutral (e.g. 'desk' and 'horse'). Given that participants were asked to make a judgement about the size of each object (see below), highly familiar and easily imaginable words were chosen. The order in which word-image pairs were presented was fully randomised.

\section{Procedure}

The study comprised one single-blind experimental session and a delayed recall session which occurred 24 hours later (24-hour recall session). The experimental session consisted of an encoding phase, a 90-minute retention interval in which participants slept in the sleep laboratory, and an immediate (90-minute) recall session (see Figure 1). At the beginning of the experimental session, participants were set up for polysomnography (PSG) recording and tACS. Following this, demographic information was recorded, and participants completed the Stanford Sleepiness Scale (Hoddes, Zarcone, Smythe, Phillips, \& Dement, 1973) which is a standard measure of subjective alertness. Participants were informed that their memory performance would be tested during the recall sessions.

\begin{tabular}{ccccc}
\hline Encoding & Sleep & Free Recall & Retention Interval & Free Recall \\
\hline tACS & PSG + tACS & & & \\
\hline $5 \mathrm{~min} 6 \mathrm{~min}$ & $90 \mathrm{~min}$ & $7 \mathrm{~min}$ & $24 \mathrm{hr}$ & $7 \mathrm{~min}$
\end{tabular}

Figure 1. Procedure of the study.

The behavioural task began 5 minutes after the onset of tACS in order to allow the stimulation to become effective. During encoding, participants were sequentially presented with 80 word-image pairs. Participants were first asked to judge, as quickly and as accurately as possible, whether each object would fit inside a conventional shoebox, and then they memorised each object for later free recall. The timeline for the encoding phase of one stimulus is displayed in Figure 2. The size judgement task was designed to ensure that participants' attention was maintained for the primary task of memorisation. 


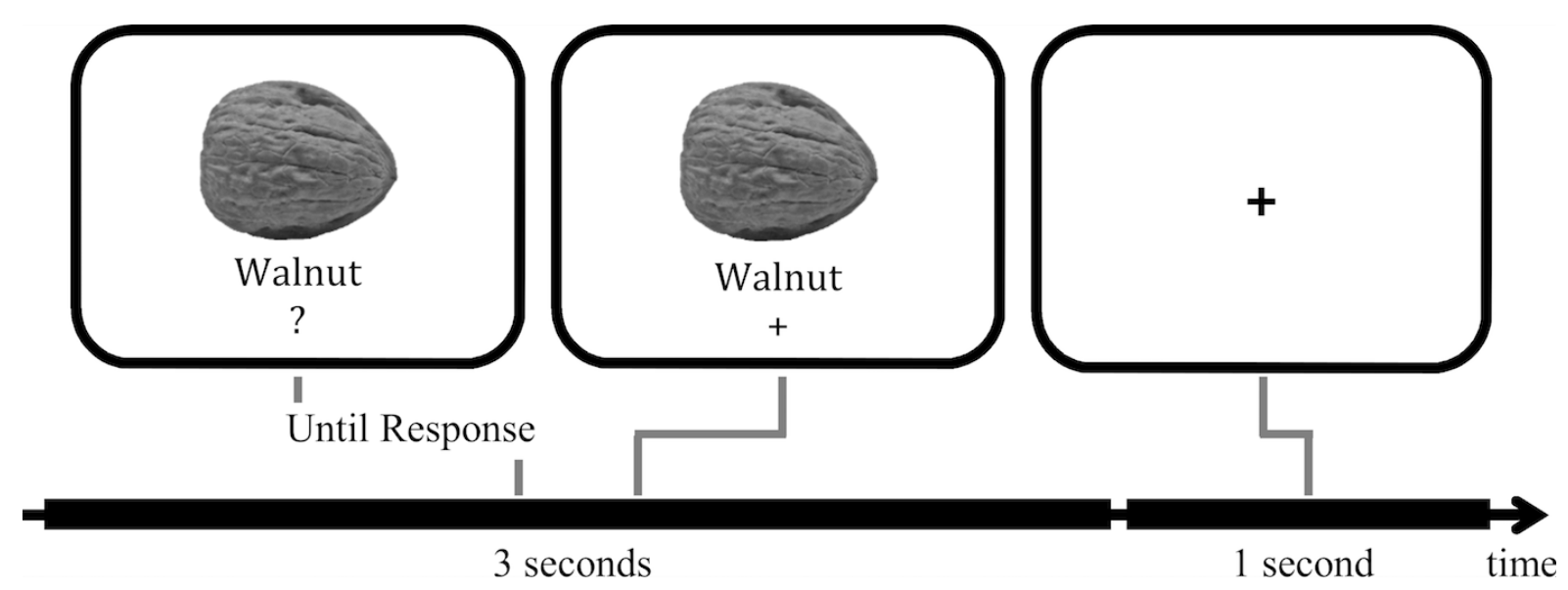

Figure 2. Timeline of encoding phase. A question mark was first presented below each word-image pair to indicate that participants should quickly and accurately respond to the size of the object using a key-press with their right hand. After participants had made their size judgement, the question mark was replaced with a small cross indicating a memorisation period. Participants were exposed to each word-image pair for 3 seconds in total. The inter-stimulus interval was indicated by a large, central fixation cross which was presented for 1 second.

After the encoding phase, there was a 90-minute retention interval in which participants slept in the sleep laboratory. Whilst participants were asleep, their PSG recording was monitored online in order to identify different sleep stages, and they received tACS according to the stimulation condition to which they had been randomly assigned. Immediately after waking and 24 hours later, participants performed a recall session in which they had 7 minutes to freely recall as many objects as possible. Participants were not given any feedback on their performance.

All stimuli were displayed on a 17-inch monitor with $60 \mathrm{~Hz}$ refresh rate. Stimulus presentation and response recording was conducted on a PC computer running MATLAB (v2013b; MathWorks Company, Natick, MA) and the Psychophysics Toolbox (v3; Brainard, 1997). Participants were seated comfortably, approximately $50 \mathrm{~cm}$ from the computer.

\section{Transcranial alternating current stimulation (tACS)}

tACS was administered by two saline-soaked surface sponge electrodes using a NeuroConn Brain Stimulator (NeuroConn, Germany). One of the electrodes $\left(3 \times 3 \mathrm{~cm}^{2}\right)$ was positioned over the left DLPFC (F3 according to the 10-20 international system for EEG electrode placement; Herwig, Satrapi, \& Schönfeldt-Lecuona, 2003; Homan, Herman, \& Purdy, 1987). The left DLPFC was targeted for stimulation because previous research has 
shown that not only is declarative memory performance associated with activity in this region, but also declarative memory performance is modulated by electrical brain stimulation to this region (Blumenfeld \& Ranganath, 2007; Cabeza \& Nyberg, 2000; Javadi \& Cheng, 2013; Javadi \& Walsh, 2012; Manenti, Brambilla, Petesi, Ferrari, \& Cotelli, 2013). The reference electrode $\left(5 \times 5 \mathrm{~cm}^{2}\right)$ was positioned on the right wrist.

During encoding, all participants received 11 minutes of tACS administered at $60 \mathrm{~Hz}$. Stimulation during sleep consisted of two, two-minute episodes of tACS administered at either $60 \mathrm{~Hz}$ or $90 \mathrm{~Hz}$ depending on the stimulation condition to which the participant had been randomly assigned. Sham stimulation sessions during sleep consisted of no stimulation. See Table 1 for a summary of stimulation conditions. During both encoding and sleep, tACS was administered with $1.5 \mathrm{~mA}$ peak-to-peak amplitude and 1-second fade in/out.

tACS was administered using two gamma frequencies $(60 \mathrm{~Hz}$ and $90 \mathrm{~Hz})$ because neural oscillations in different frequency bands have different roles in memory function (for reviews, see Başar, Başar-Eroglu, Karakaş, \& Schürmann, 2001; Düzel, Penny, \& Burgess, 2010; Lisman, 2010). Therefore, in order to investigate oscillatory reinstatement without the confounding effect of administering tACS in different frequency bands, the current study used different frequencies from the same band. Importantly, the gamma band has a wide enough frequency range $(+30 \mathrm{~Hz})$ in order for two frequencies (e.g. $60 \mathrm{~Hz}$ and $90 \mathrm{~Hz}$ ) to be selected that are sufficiently distinct from each other, such that administering tACS at one frequency will have a minimal effect at the other frequency.

\section{Polysomnography (PSG)}

Sleep was recorded by standard PSG (Rechtschaffen \& Kales, 1968) using a 32channel, eego sports system (ANT Neuro, Germany). Electroencephalography (EEG) was recorded from twenty-nine scalp electrodes which were positioned using the 10-20 international system for EEG electrode placement (Herwig et al., 2003; Homan et al., 1987). Electrode F3 was not recorded due to tACS electrode placement.

In order to ascertain the accuracy of tACS administration according to stimulation conditions and to extract sleep parameters (e.g. duration of SWS and REM sleep), the PSG data recorded during sleep were reanalysed after the experimental session. EEG signals were band-pass filtered between $0.5-30 \mathrm{~Hz}$ and downsampled to $128 \mathrm{~Hz}$, montaged based on average activity. Sleep duration data were obtained by scoring 20-second epochs of PSG recordings offline into wake, non-REM stage 1, non-REM stage 2, non-REM stage 3 (SWS), 
and REM sleep, according to the AASM scoring manual v2.2 (Berry et al., 2016). Offline sleep scoring was performed using the sleepSMG package (http://sleepsmg.sourceforge.net) for MATLAB. Epochs during the stimulation were marked as unknown.

\section{Statistical data analysis}

To assess the effect of tACS administered during encoding and sleep on post-sleep declarative memory performance, a $2 \times 4$ mixed analysis of variance (ANOVA) was conducted. Recall session (90-minute recall session and 24-hour recall session) was the within-participants factor, and stimulation condition (congruent-SWS, incongruent-SWS, congruent-REM, and sham) was the between-participants factor. The dependent variable was the number of words correctly recalled. A similar ANOVA was conducted with the number of words falsely recalled as the dependent variable. Five separated one-way ANOVAs were conducted with stimulation condition as the between-participants factor, and sleep parameters (durations of total sleep, Stage 1, Stage 2, SWS, and REM sleep) as the dependent variables. Post-hoc two independent sample t-tests were used to investigate differences in memory recall between stimulation conditions. Partial-eta squared is the effect size reported for ANOVA.

To investigate whether relationships exist between the durations of specific sleep stages and the extent of sleep-dependent declarative memory consolidation in each stimulation condition, correlational analyses were conducted between time spent in each sleep stage and correct memory recall during the 90-minute and 24-hour recall sessions. Correlations were calculated using Spearman's rho correlations. All statistical analyses on the data were conducted using SPSS (v21; IBM Lead Technologies, Inc.).

\section{Results}

This study investigated whether oscillatory reinstatement enhances sleep-dependent declarative memory consolidation, by assessing declarative memory performance during two recall sessions which occurred after tACS had been administered to participants' left DLPFC at either the same or different gamma frequency during encoding and sleep. The ANOVA analysing number of words correctly recalled between stimulation conditions and recall sessions revealed a significant main effect of recall session $\left(F(1,78)=63.79, p<.001, \eta_{p}{ }^{2}=\right.$ $.45)$, a significant main effect of stimulation condition $\left(F(3,78)=4.03, p=.01, \eta_{p}{ }^{2}=.13\right)$, 
and no significant interaction effect $\left(F(3,78)=1.36, p=.26, \eta_{p}{ }^{2}=.05\right)$. Post-hoc two independent sample t-tests were conducted to compare memory recall between stimulation conditions. In line with the initial hypotheses, post-hoc t-tests revealed that during both the 90-minute and 24-hour recall sessions, participants in the congruent-SWS stimulation condition correctly recalled significantly more words than participants in any other stimulation condition (incongruent-SWS, congruent-REM, and sham; see Table 2). Hence, oscillatory reinstatement during SWS facilitated sleep-dependent declarative memory consolidation, and thus enhanced post-sleep declarative memory performance for participants in this stimulation condition. Notably, although learning performance during training was not assessed, it can be assumed from the large sample size that baseline memory performance was comparable between groups before sleep.

The ANOVA analysing number of words falsely recalled showed no significant effects $(p s>.09)$. A summary of the descriptive data for memory recall according to stimulation condition and recall session is displayed in Table 3 and Figure 3. The five separate ANOVAs analysing sleep parameters showed no significant differences between stimulation groups $(p s>.10)$.

Table 2. Summary of post-hoc two independent sample $t$ tests for number of words correctly recalled between stimulation conditions.

\begin{tabular}{ll}
\hline Comparison & $p$ \\
\hline Congruent-SWS vs. Incongruent-SWS & $.002^{*}$ \\
Congruent-SWS vs. Congruent-REM & $.025^{*}$ \\
Congruent-SWS vs. Sham & $.007^{*}$ \\
\hline note: ${ }^{*} p<.05$ &
\end{tabular}


Table 3. Mean and standard deviation of words correctly and falsely recalled during the 90minute recall session and the 24-hour recall session for participants in each stimulation condition.

\begin{tabular}{llll}
\hline Recall Session & Condition & Correct Recall & False Recall \\
\hline 90-minute & Congruent-SWS & $26.60[5.63]$ & $1.40[1.43]$ \\
& Incongruent-SWS & $20.53[7.32]$ & $2.29[2.11]$ \\
& Congruent REM & $22.78[6.51]$ & $1.56[1.79]$ \\
\multirow{2}{*}{ 24-hour } & Sham & $22.48[6.42]$ & $1.85[1.70]$ \\
& Congruent-SWS & $24.25[7.73]$ & $1.95[2.01]$ \\
& Incongruent-SWS & $16.71[6.93]$ & $2.24[2.28]$ \\
& Congruent REM & $18.44[7.45]$ & $1.11[1.45]$ \\
& Sham & $17.67[6.84]$ & $1.37[1.36]$ \\
\hline
\end{tabular}

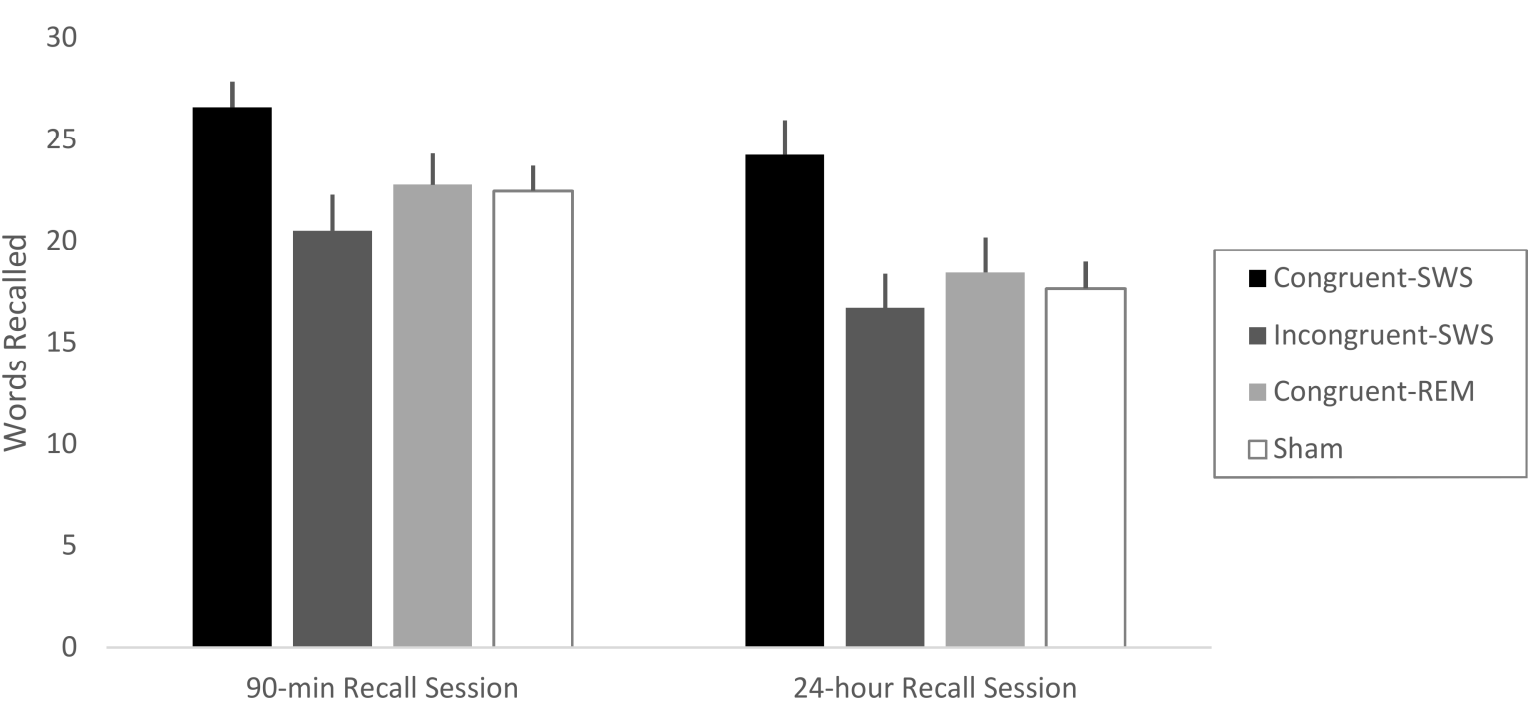

Figure 3. Mean number of words correctly recalled during the 90-minute recall session and the 24-hour recall session for participants in each stimulation condition. Error bars represent one standard error.

This study also investigated whether the durations of specific sleep stages correlate with the extent of sleep-dependent declarative memory consolidation in each stimulation condition. Spearman's rho correlations revealed that for each stimulation condition, there were no significant correlations of interest. See Table 4 for the average time spent in each sleep stage for each stimulation condition. note: $p>0.07$ for all comparisons between stimulation conditions and sleep parameters 
Table 5 displays a summary of all correlations between time spent in each sleep stage and correct memory recall during the 90-minute recall session and the 24-hour recall session.

Table 4. Average time spent in each sleep stage for each stimulation condition. Time is reported in minutes (mean $[\mathrm{SD}]$ ).

\begin{tabular}{llllll}
\hline Condition & Sleep & Stage 1 & Stage 2 & SWS & REM \\
\hline Congruent-SWS & $72.75[15.14]$ & $10.73[6.47]$ & $26.40[9.17]$ & $26.07[13.08]$ & $5.55[7.30]$ \\
Incongruent-SWS & $73.27[11.66]$ & $13.78[7.34]$ & $25.31[9.19]$ & $25.06[13.70]$ & $5.35[7.69]$ \\
Congruent REM & $72.83[11.99]$ & $14.50[7.23]$ & $29.74[7.82]$ & $15.24[12.67]$ & $9.63[7.88]$ \\
Sham & $69.27[17.62]$ & $15.88[11.34]$ & $27.02[11.64]$ & $15.73[13.18]$ & $5.64[7.64]$ \\
\hline
\end{tabular}

note: $p>0.07$ for all comparisons between stimulation conditions and sleep parameters

Table 5. Correlations between time spent in each sleep stage and correct memory recall during the 90-minute recall session and the 24-hour recall session.

\begin{tabular}{|c|c|c|c|c|c|c|c|c|c|c|}
\hline \multirow[b]{2}{*}{ Condition } & \multicolumn{2}{|c|}{ Total Sleep } & \multicolumn{2}{|c|}{ Stage 1} & \multicolumn{2}{|c|}{ Stage 2} & \multicolumn{2}{|l|}{ SWS } & \multicolumn{2}{|l|}{ REM } \\
\hline & $r$ & $p$ & $r$ & $p$ & $r$ & $p$ & $r$ & $p$ & $\boldsymbol{r}$ & $p$ \\
\hline \multicolumn{11}{|l|}{ 90-minute } \\
\hline Congruent-SWS & 0.14 & 0.56 & 0.32 & 0.17 & 0.09 & 0.72 & -0.23 & 0.33 & 0.39 & 0.09 \\
\hline Incongruent-SWS & -0.07 & 0.78 & -0.48 & $0.05^{\dagger}$ & 0.18 & 0.49 & 0.02 & 0.93 & 0.4 & 0.11 \\
\hline Congruent REM & 0.62 & $0.01^{*}$ & -0.48 & $0.05^{\dagger}$ & 0.23 & 0.36 & 0.47 & $0.05^{\dagger}$ & 0.33 & 0.18 \\
\hline Sham & 0.13 & 0.52 & 0.10 & 0.64 & 0.08 & 0.69 & 0.23 & 0.24 & -0.24 & 0.24 \\
\hline \multicolumn{11}{|l|}{ 24-hour } \\
\hline Congruent-SWS & 0.01 & 0.97 & 0.39 & 0.09 & -0.01 & 0.97 & -0.44 & $0.05^{\dagger}$ & 0.36 & 0.12 \\
\hline Incongruent-SWS & -0.02 & 0.94 & -0.44 & $0.05^{\dagger}$ & 0.26 & 0.31 & 0.04 & 0.88 & 0.33 & 0.19 \\
\hline Congruent REM & 0.43 & 0.08 & -0.42 & 0.08 & 0.37 & 0.13 & 0.32 & 0.19 & 0.23 & 0.36 \\
\hline Sham & 0.04 & 0.83 & 0.15 & 0.46 & 0.08 & 0.68 & 0.09 & 0.66 & -0.29 & 0.14 \\
\hline
\end{tabular}

note: ${ }^{*} p<0.05,{ }^{\dagger} p=0.05$

\section{Discussion}

To investigate whether oscillatory reinstatement facilitates the engram reactivation that underlies sleep-dependent memory consolidation, the current study assessed participants' 
declarative memory performance after they had received tACS during encoding and postlearning sleep. The results found that administering tACS to the left DLPFC has a modulatory effect on subsequent post-sleep declarative memory performance, and this effect was specific to the stimulation frequency and sleep stage in which tACS was administered. With regards to frequency specificity, memory recall was greater during immediate and delayed recall sessions if participants had been administered with the same frequency of stimulation during encoding and sleep, compared to if they had been administered with different frequencies or sham stimulation. Crucially, to the best of our knowledge, no study has demonstrated a functional difference between gamma frequencies (e.g. $60 \mathrm{~Hz}$ and $90 \mathrm{~Hz}$ ) in relation to memory mechanisms or brain stimulation (see Düzel et al., 2010). Therefore, it is unlikely that this result was caused by a general effect of administering tACS during SWS at $60 \mathrm{~Hz}$ rather than $90 \mathrm{~Hz}$. With regards to sleep stage specificity, the results found that for participants who received congruent stimulation during encoding and sleep, memory recall was greater if SWS was the sleep stage that had been targeted compared to if REM sleep had been targeted. However, the durations of specific sleep stages did not correlate with postsleep declarative memory performance, and there were no significant differences in sleep parameters between stimulation conditions. Notably, in line with a robust finding in the memory literature that memory recall decreases as the duration of a retention interval increases (Altmann \& Gray, 2002; Brown, 1958; Keppel \& Underwood, 1962; Peterson \& Peterson, 1959; Ricker \& Cowan, 2010; for a review of the time-related decay vs. interference-based forgetting debate, see Jonides et al., 2008), the results also found that memory recall was greater during the immediate recall session compared to the delayed recall session.

Previous research found evidence to support SCERT's prediction that since frequency-specific oscillatory activity coordinates PS and CFC, which are neural mechanisms that contribute to engram reactivation, memory recall will be enhanced when the same frequency of neural oscillations that occurred during encoding is reinstated during retrieval (Javadi et al., 2017; Watrous \& Ekstrom, 2014). Additionally, evidence supports the assumption of the active system consolidation hypothesis that engram reactivation has a fundamental role in the redistribution process that underlies sleep-dependent memory consolidation (Marshall \& Born, 2007; Peigneux et al., 2004; Rasch et al., 2007). Therefore, the current finding that declarative memory performance is enhanced following the reinstatement of frequency-specific oscillatory activity between encoding and post-learning 
SWS has integrated and furthered these previous lines of research. More specifically, this finding demonstrates that by experimentally inducing oscillatory reinstatement, it is possible to facilitate the engram reactivation that underlies sleep-dependent memory consolidation as well as the engram reactivation that underlies memory retrieval. Moreover, the current finding is in line with ample research suggesting that SWS, rather than REM sleep, is responsible for declarative memory consolidation (Maquet, 2001; Pilhal \& Born, 1997, 1999; Rasch et al., 2007).

Importantly, these results have considerable implications for the advancement of theories within the sleep-dependent memory consolidation literature. If sleep-dependent declarative memory consolidation can be modulated by experimentally-induced oscillatory reinstatement, it seems that typical sleep-dependent declarative memory consolidation must be dependent on an intrinsic neurobiological mechanism for oscillatory reinstatement. In fact, this suggestion builds on the assumptions and supporting evidence of SCERT, which have already recognised the importance of an intrinsic mechanism for oscillatory reinstatement during memory retrieval. In contrast, whereas ample research has implicated various roles for specific frequencies of neural oscillations in sleep-dependent memory consolidation (Buzsáki, 1998; Ladenbauer et al., 2017; Marshall, Helgadóttir, Mölle, \& Born, 2006; Marshall, Kirov, Brade, Mölle, \& Born, 2011; Nitsche et al., 2010; for review, see Barham, Enticott, Conduit, \& Lum, 2016; Rasch \& Born, 2013), no theory of memory consolidation has, as yet, delineated a role for oscillatory reinstatement specifically. Consequently, the current results provide causal evidence in support of a novel theoretical proposal, which assumes that sleep-dependent declarative memory consolidation is underpinned by an intrinsic neurobiological mechanism that coordinates oscillatory activity, during SWS, according to activity that occurred during encoding.

Although it is proposed that an intrinsic neurobiological mechanism for oscillatory reinstatement has a fundamental role in typical sleep-dependent declarative memory consolidation, the neural correlate of this mechanism remains unclear. On the one hand, the current modulatory effect of oscillatory reinstatement was found after administering tACS to the left DLPFC - a brain region that has previously been implicated in declarative memory consolidation (Blumenfeld \& Ranganath, 2007; Cabeza \& Nyberg, 2000; Javadi \& Cheng, 2013). Therefore, it seems plausible that the left DLPFC would also be the locus of intrinsic frequency-specific oscillatory activity during sleep-dependent declarative memory consolidation. Moreover, this brain region is highly connected with cortical and subcortical 
structures, and it has multiple feedforward projections for neurotransmission (Petrides \& Pandya, 1999; Ray \& Zald, 2012). Hence, the DLPFC also has structural connectivity that would support the distribution of frequency-specific oscillatory activity to multiple brain regions. On the other hand, however, it is important to note that electrical brain stimulation is relatively diffuse, such that brain regions adjacent to a target site are often stimulated (Bikson, Rahman, \& Datta, 2012). Consequently, perhaps the current finding actually occurred because the intrinsic mechanism for oscillatory reinstatement is located in a brain region which is spatially proximal to the left DLPFC, and thus was also stimulated by tACS administered to the left DLPFC. Therefore, in order to locate the neural correlate of this intrinsic mechanism more precisely, future research should administer experimentallyinduced oscillatory reinstatement to various scalp locations, and assess the location specificity of any subsequent modulation to sleep-dependent declarative memory consolidation.

Alternatively, it is possible that even if the current finding was caused by stimulation of the left DLPFC specifically, rather than of adjacent brain regions, the left DLPFC may still not be the locus of intrinsic oscillatory activity during sleep-dependent declarative memory consolidation. The reason for this suggestion is that the hippocampus is one of the cortical structures that has ample structural connections, both directly and indirectly, with the DLPFC (Ding, 2013; Goldman-Rakic, Selemon, \& Schwartz, 1984; Schott et al., 2011). Importantly, the hippocampus is a suitable candidate for the neural correlate of an intrinsic oscillatory reinstatement mechanism, because this region is consistently implicated in processing the spatiotemporal contexts of stimuli (for reviews, see Burgess, Maguire, \& O’Keefe, 2002; Eichenbaum, 2004; Nadel \& Moscovitch, 1997; Teyler \& DiScenna, 1985; Teyler \& Rudy, 2007). Consequently, it seems likely that the hippocampus would have a role in coordinating the frequency-specific oscillatory activity that constitutes the implicit neural contexts of stimuli. In fact, this suggestion is particularly feasible in light of findings that hippocampal regions encode spatial and temporal information in a frequency-specific manner (Watrous, Tandon, Connor, Pieters, \& Ekstrom, 2013). Perhaps, therefore, sleep-dependent declarative memory consolidation was modulated by stimulation of the left DLPFC in the current study, because a hippocampally-mediated mechanism for intrinsic oscillatory reinstatement was stimulated as a result of the structural connections between the left DLPFC and the hippocampus. Notably, this proposal is in line with SCERT which posits that the hippocampus has the necessary structural organisation and circuity to induce oscillatory 
reinstatement intrinsically during memory retrieval (Watrous \& Ekstrom, 2014). Future research should employ deep brain stimulation techniques to further determine whether intrinsic frequency-specific oscillatory activity originates in the hippocampus during sleepdependent declarative memory consolidation (see Fell et al., 2013).

Despite this, it could also be argued that the current finding is a result of functional, rather than structural connections, with the left DLPFC. Ample evidence demonstrates that the DLPFC forms a functional network with the ventrolateral prefrontal cortex (VLPFC), whereby the left VLPFC is responsible for declarative memory encoding and the left DLPFC is responsible for subsequent organisation and retrieval processes (Blumenfeld, Lee, \& D’Esposito, 2014; Galli, Feurra, Pavone, Sirota, \& Rossi, 2017; Medvedeva et al., 2018; for reviews, see Blumenfeld \& Ranganath, 2007; Fletcher \& Henson, 2001; Nyhus \& Badre, 2015). Notably, the VLPFC and the DLPFC are thought to coordinate their activity via neural oscillations (Nyhus \& Badre, 2015). Therefore, since the left VLPFC is responsible for encoding, it could be that intrinsic frequency-specific oscillatory activity is generated in this region before it is subsequently projected to the left DLPFC, during SWS, to facilitate consolidation. In this case, the current finding may have occurred because, by administering a congruent frequency of stimulation during encoding and SWS, the stimulation protocol facilitated oscillatory activity that was being intrinsically projected from the left VLPFC to the left DLPFC. Henceforth, although sleep-dependent declarative memory consolidation can be modulated by experimentally-induced oscillatory reinstatement in the left DLPFC, the left VLPFC may be the brain region in which intrinsic frequency-specific oscillatory activity originates. If future research were to inhibit projections from the left VLPFC during sleepdependent declarative memory consolidation, it may be possible to determine whether this brain region is the neural correlate of an intrinsic oscillatory reinstatement mechanism.

Further to this, the implications of the current results include practical contributions, as well as theoretical contributions, to the literature. This claim is based on the finding that participants, who received congruent stimulation during encoding and SWS, demonstrated a declarative memory enhancement that was maintained after an additional night of sleep. Theoretically, this finding indicates that the faciliatory effect of experimentally-induced oscillatory reinstatement on sleep-dependent declarative memory consolidation is additive, such that further overnight processing cannot mediate the enhancement. Notably, this opposes previous research which asserted that sleep intervention-related memory enhancements are short-term, because control participants demonstrate delayed consolidation 
effects after additional sleep, which attenuate differences in memory performance between control and experimental conditions (Shimizu et al., 2018). Henceforth, the persistence of the current declarative memory enhancement highlights the importance of oscillatory reinstatement for sleep-dependent declarative memory consolidation. In terms of practical contributions, this finding suggests that non-invasive brain stimulation protocols could be used in therapeutic settings to elicit memory enhancements that are long-term. For example, mild cognitive impairment (MCI) is a non-degenerative condition characterised by impaired memory, language, and sleep physiology (Petersen et al., 2014; Roark, Mitchell, Hosom, Hollingshead, \& Kaye, 2011; Westerberg et al., 2012). Therefore, if MCI patients were administered with experimentally-induced oscillatory reinstatement during encoding and SWS, it might be possible to elicit additive, long-term enhancements in their declarative memory which is critical for everyday living, but which is disrupted by the condition (see Ladenbauer et al., 2017).

Notably, although the current results support the assumption that declarative memory consolidation relies on SWS more than REM sleep, it is important to note that the occurrence of REM sleep may still have been necessary for these results. The sequential hypothesis of sleep-dependent memory consolidation assumes that memory consolidation relies on the cyclic succession of SWS and REM sleep because, following destabilisation during reactivation in SWS, engrams must be reconsolidated during REM sleep (Gais, Pilhal, Wagner, \& Born, 2000; Mednick, Nakayama, \& Stickgold, 2003; for reviews, see Ambrosini \& Giuditta, 2001; Diekelmann \& Born, 2010; Giuditta et al., 1995). In line with this, studies using TMR to reinstate contextual cues between encoding and SWS, found that post-sleep memory performance for cued stimuli correlated positively with REM sleep duration (Cousins et al., 2016; Tamminen et al., 2017). Hence, increased engram reactivation (and thus destabilisation) during SWS was more able to facilitate post-sleep memory performance when it was succeeded by increased reconsolidation during REM sleep. Importantly, although post-sleep declarative memory performance did not correlate with REM sleep duration in the current study, this result may simply have been caused by the methodology. The reason being that whereas previous studies re-presented contextual cues during sleep that do not affect sleep architecture, such as odours (Carskadon \& Herz, 2004), the current study administered electrical brain stimulation which is known to alter sleep parameters (Frase et al., 2016; Marshall et al., 2011; for review, see Rivera-Urbina, Nitsche, \& Molero-Chamizo, 2016). Moreover, since the current study employed a nap paradigm rather than an overnight 
paradigm, there was a limited duration of sleep in which sleep parameters could actually be assessed (Ackermann \& Rasch, 2014; Hobson \& Pace-Schott, 2002), Consequently, the current lack of sleep parameter correlations is unsurprising, and does not oppose a role for both SWS and REM sleep in declarative memory consolidation.

A potential criticism of the current study relates to the fact that neural activation following electrical brain stimulation was not monitored using a neuroimaging technique that provides volumetric images of brain activity. Consequently, the current study was unable to obtain neural evidence supporting the central premise of the current conclusion; that oscillatory reinstatement facilitates engram reactivation during sleep-dependent declarative memory consolidation. Notably, previous studies (e.g. Cousins et al., 2016; Rasch et al., 2007) used functional magnetic resonance imaging (fMRI) to confirm the occurrence of engram reactivation after reinstating explicit environmental contexts between encoding and SWS. Therefore, future research should use fMRI to strengthen the current conclusions by providing neural evidence demonstrating that engram reactivation is elicited by oscillatory reinstatement during SWS. Alternatively, future research could strengthen the current conclusions by investigating correlations between declarative memory performance following oscillatory reinstatement and sleep spindle activity. Importantly, sleep spindles are considered to be markers of engram reactivation during sleep-dependent memory consolidation, because they are temporally correlated with oscillatory events in both the hippocampus (sharp wave ripples) and the neocortex (slow oscillations; Antony et al., 2018; Helfrich, Mander, Jagust, Knight, \& Walker, 2018; Mölle, Marshall, Gais, \& Born, 2002; Staresina et al., 2015; for reviews, see Diekelmann, \& Born, 2010; O’Neill et al., 2010; Rasch \& Born, 2013). Hence, if oscillatory reinstatement enhances sleep-dependent declarative memory consolidation by facilitating engram reactivation, number of sleep spindles following oscillatory reinstatement might be expected to correlate positively with post-sleep declarative memory performance (see also Ladenbauer et al., 2016; Paßmann et al., 2016). Notably, the current study did not assess sleep parameters, such as spindle activity or slow-wave activity, following brain stimulation because the experimental design meant that stimulation was sometimes administered at the end of the sleep period. In contrast, an overnight study would allow for investigating effects of brain stimulation on sleep parameters in subsequent sleep cycles.

Future research should explore the scope of the role of oscillatory reinstatement in sleep-dependent memory consolidation, and the following two lines of research are 
recommended in order to pursue this objective. Firstly, the robustness of the current findings should be established in terms of stimulation frequency and task modality. With regards to stimulation frequency, it has been shown that although neural oscillations in both the gamma and theta frequency bands are implicated in memory processing, the two frequency bands have different functional roles (Osipova et al., 2006; Sederberg, Kahana, Howard, Donner, \& Madsen, 2003; von Stein \& Sarnthein, 2000; for reviews, see Başar et al., 2001; Düzel, et al., 2010; Hanslmayr \& Staudigl, 2014; Nyhus \& Curran, 2010). Therefore, it would be useful to determine whether experimentally-induced oscillatory reinstatement during SWS can still enhance post-sleep declarative memory performance when theta frequencies are stimulated instead of gamma frequencies. Additionally, future research could explore the strength of the current oscillatory reinstatement effect by administering tACS in frequency bands that are yet to be strongly implicated in memory mechanisms.

With regards to task modality, there is evidence suggesting that the modulatory effect of oscillatory reinstatement on memory retrieval is influenced by task modality at encoding and retrieval (Staudigl \& Hanslmayr, 2018). In Staudigl and Hanslmayr's (2018) study, participants learned visually- or auditorily-presented words, and performed a subsequent recognition task in which the cue words were presented visually or auditorily. The results found that if task modalities were congruent between encoding and retrieval, oscillatory reinstatement between encoding and retrieval enhanced memory performance, whereas oscillatory reinstatement impaired memory performance if task modalities were incongruent. Therefore, future research should investigate whether the modulatory effect of oscillatory reinstatement on sleep-dependent memory consolidation is also influenced by task modality. These results would have implications for the extent to which the current stimulation protocol could be used to elicit declarative memory enhancements in real-life settings where task modalities are less easily controlled.

Secondly, future research should extend the current findings by assessing the role of oscillatory reinstatement in the sleep-dependent consolidation of non-declarative memories. On the one hand, if the hippocampus is found to be the neural correlate of an intrinsic mechanism for oscillatory reinstatement, experimentally-induced oscillatory reinstatement might be unable to enhance post-sleep non-declarative memory performance because these memories are consolidated independently of the hippocampus (for reviews, see Cabeza \& Moscovitch, 2013; Squire, 1992; Squire \& Zola, 1996; see also Barham et al., 2016). On the other hand, since REM sleep, rather than SWS, is responsible for non-declarative memory 
consolidation, there could be a different intrinsic mechanism for coordinating oscillatory activity during REM sleep. Therefore, perhaps, experimentally-induced oscillatory reinstatement would be able to enhance non-declarative memory consolidation if this mechanism were stimulated during REM sleep. Despite this, if future research were to demonstrate enhanced post-sleep non-declarative memory performance following experimentally-induced oscillatory reinstatement during REM sleep, this finding would not necessarily support the current conclusion that increased engram reactivation underlies this effect. The reason being that non-declarative memory consolidation depends on synaptic plasticity which is supported by molecular and electrophysiological events during REM sleep (for review, see Diekelmann \& Born, 2010). Crucially, synaptic plasticity is facilitated by the neural mechanisms - PS and CFC - which also facilitate engram reactivation, and which are coordinated by frequency-specific oscillatory activity (for reviews, see Canolty \& Knight, 2010; Fell \& Axmacher, 2011; Jutras and Buffalo, 2010). Therefore, the potential effect of experimentally-induced oscillatory reinstatement during REM sleep on post-sleep nondeclarative memory performance could be caused by either increased synaptic plasticity or increased engram reactivation. Henceforth, future research should also distinguish between these interpretations.

To conclude, the current study revealed that by using electrical brain stimulation to reinstate the same frequency of neural oscillations between encoding and slow-wave sleep, post-sleep declarative memory performance can be enhanced. This finding furthers SCERT by demonstrating that oscillatory reinstatement facilitates the engram reactivation that underlies sleep-dependent memory consolidation, as well as that which underlies memory retrieval. Moreover, this finding provides support for a novel theoretical proposal, which assumes that an intrinsic neurobiological mechanism for coordinating frequency-specific oscillatory activity, during SWS, underlies sleep-dependent declarative memory consolidation. Although the current study did not identify the neural correlate of this intrinsic mechanism, several candidate brain regions have been considered, along with recommendations for future studies to investigate these suggestions. Additionally, several directions for future research have been proposed which would not only strengthen support for the current conclusions, but would also assess the scope of the role of oscillatory reinstatement in sleep-dependent memory consolidation more generally. Finally, the current study has succeeded in introducing a novel non-invasive brain stimulation protocol, which has the potential to be used therapeutically for enhancing declarative memory performance in 
patient populations. Henceforth, the current findings indicate that the memory literature would benefit from directing more emphasis towards the importance of oscillatory reinstatement in memory function.

\section{Acknowledgements}

Authors are especially grateful to Juliana Sporrer for assisting with data collection, and to Susanne Diekelmann and Penny Lewis for their invaluable comments on an earlier draft of this manuscript.

\section{References}

Ackermann, S., \& Rasch, B. (2014). Differential effects of non-REM and REM sleep on memory consolidation? Current Neurology and Neuroscience Reports, 14(2), 430440. doi: 10.1007/s 11910-013-0430-8.

Altmann, E. M., \& Gray, W. D. (2002). Forgetting to remember: The functional relationship of decay and interference. Psychological Science, 13(1), 27-33. doi: 10.1111/14679280.00405 .

Ambrosini, M. V., \& Giuditta, A. (2001). Learning and sleep: The sequential hypothesis. Sleep Medicine Reviews, 5(6), 477-490. doi: 10.1053/smrv.2001.0180.

Antal, A., \& Paulus, W. (2013). Transcranial alternating current stimulation (tACS). Frontiers in Human Neuroscience, 7(317), 1-4. doi: 10.3389/fnhum. 2013.00317.

Antony, J. W., Gobel, E. W., O'Hare, J. K., Reber, P. J., \& Paller, K. A. (2012). Cued memory reactivation during sleep influences skill learning. Nature Neuroscience, 15(8), 1114-1116. doi: 10.1038/nn.3152.

Antony, J. W., Piloto, L., Wang, M., Pacheco, P., Norman, K. A., \& Paller, K. A. (2018). Sleep spindle refractoriness segregates periods of memory reactivation. Current Biology, 28(11), 1736-1743. doi: 10.1016/j.cub.2018.04.020.

Ashton, J. E., Cairney, S. A., \& Gaskell, M. G. (2018). No effect of targeted memory reactivation during slow-wave sleep on emotional recognition memory. Journal of Sleep Research, 27(1), 129-137. doi: 10.1111/jsr.12542. 
Barham, M. P., Enticott, P. G., Conduit, R., \& Lum, J. A. (2016). Transcranial electrical stimulation during sleep enhances declarative (but not procedural) memory consolidation: Evidence from a meta-analysis. Neuroscience \& Biobehavioral Reviews, 63, 65-77. doi: 10.1016/j.neubiorev.2016.01.009.

Başar, E., Başar-Eroglu, C., Karakaş, S., \& Schürmann, M. (2001). Gamma, alpha, delta, and theta oscillations govern cognitive processes. International Journal of Psychophysiology, 39(2-3), 241-248. doi: 10.1016/S0167-8760(00)00145-8.

Berry, R. B., Brooks, R., Gamaldo, C. E., Harding, S. M., Lloyd, R, M., Marcus, C. L., \& Vaughn, B. V. (2016). The AASM manual for the scoring of sleep and associated events: Rules, terminology, and technical specifications, version 2.2. American Academy of Sleep, 28(3), 391-397. doi: 10.1017/CBO9781107415324.004.

Bikson, M., Rahman, A., \& Datta, A. (2012). Computational models of transcranial direct current stimulation. Clinical EEG and Neuroscience,43(3), 176-183. doi: $10.1177 / 1550059412445138$.

Blumenfeld, R. S., Lee, T. G., \& D’Esposito, M. (2014). The effects of lateral prefrontal transcranial magnetic stimulation on item memory encoding. Neuropsychologia, 53, 197-202. doi: 10.1016/j.neuropsychologia.2013.11.021.

Blumenfeld, R. S., \& Ranganath, C. (2007). Prefrontal cortex and long-term memory encoding: an integrative review of findings from neuropsychology and neuroimaging. The Neuroscientist, 13(3), 280-291. doi: 10.1177/1073858407299290.

Born, J., \& Wilhelm, I. (2012). System consolidation of memory during sleep. Psychological Research, 76(2), 192-203. doi: 10.1007/s00426-011-0335-6.

Bragin, A., Jandó, G., Nádasdy, Z., Hetke, J., Wise, K., \& Buzsáki, G. (1995). Gamma (40$100 \mathrm{~Hz}$ ) oscillation in the hippocampus of the behaving rat. Journal of Neuroscience, 15(1), 47-60. doi: 10.1523/JNEUROSCI.15-01-00047.1995.

Brainard, D. H. (1997). The psychophysics toolbox. Spatial Vision, 10(4), 433-436. doi: $10.1163 / 156856897 X 00357$.

Brown, J. (1958). Some tests of the decay theory of immediate memory. Quarterly Journal of Experimental Psychology, 10(1), 12-21. doi: 10.1080/17470215808416249.

Burgess, N., Maguire, E. A., \& O'Keefe, J. (2002). The human hippocampus and spatial and episodic memory. Neuron, 35(4), 625-641. doi: 10.1016/S0896-6273(02)00830-9. 
Buzsáki, G. (1998). Memory consolidation during sleep: A neurophysiological perspective. Journal of Sleep Research,7(S1), 17-23. doi: 10.1046/j.13652869.7.s1.3.x.

Cabeza, R., \& Moscovitch, M. (2013). Memory systems, processing modes, and components: Functional neuroimaging evidence. Perspectives on Psychological Science, 8(1), 4955. doi: 10.1177/1745691612469033.

Cabeza, R., \& Nyberg, L. (2000). Imaging cognition II: An empirical review of 275 PET and fMRI studies. Journal of Cognitive Neuroscience, 12(1), 1-47. doi: $10.1162 / 08989290051137585$.

Cairney, S. A., Durrant, S. J., Hulleman, J., \& Lewis, P. A. (2014). Targeted memory reactivation during slow wave sleep facilitates emotional memory consolidation. Sleep, 37(4), 701-707. doi: 10.5665/sleep.3572.

Canavier, C. C. (2015). Phase-resetting as a tool of information transmission. Current Opinion in Neurobiology, 31, 206-213. doi: 10.1016/j.conb.2014.12.003.

Canolty, R. T., \& Knight, R. T. (2010). The functional role of cross-frequency coupling. Trends in Cognitive Sciences, 14(11), 506-515. doi: 10.1016/ j.tics.2010.09.001.

Carskadon, M. A., \& Herz, R. S. (2004). Minimal olfactory perception during sleep: Why odor alarms will not work for humans. Sleep, 27(3), 402-405. doi: 10.1093/ sleep/27.3.402.

Cousins, J. N., El-Deredy, W., Parkes, L. M., Hennies, N., \& Lewis, P. A. (2016). Cued reactivation of motor learning during sleep leads to overnight changes in functional brain activity and connectivity. PLoS Biology, 14(5), 1-21. doi: 10.1371/ journal.pbio.1002451.

Diekelmann, S., \& Born, J. (2010). The memory function of sleep. Nature Reviews Neuroscience, 11(2), 114-126. doi: 10.1038/nrn2762.

Diekelmann, S., Born, J., \& Rasch, B. (2016). Increasing explicit sequence knowledge by odor cueing during sleep in men but not women. Frontiers in Behavioral Neuroscience, 10(74), 1-11. doi: 10.3389/fnbeh.2016.00074.

Ding, S. L. (2013). Comparative anatomy of the prosubiculum, subiculum, presubiculum, postsubiculum, and parasubiculum in human, monkey, and rodent. Journal of Comparative Neurology, 521(18), 4145-4162. doi: 10.1002/cne.23416. 
Düzel, E., Penny, W. D., \& Burgess, N. (2010). Brain oscillations and memory. Current Opinion in Neurobiology, 20(2), 143-149. doi: 10.1016/j.conb.2010.01.004.

Eichenbaum, H. (2004). Hippocampus: Cognitive processes and neural representations that underlie declarative memory. Neuron,44(1), 109-120. doi: 10.1016/j.neuron. 2004.08.028.

Ellenbogen, J. M., Hulbert, J. C., Stickgold, R., Dinges, D. F., \& Thompson-Schill, S. L. (2006). Interfering with theories of sleep and memory: Sleep, declarative memory, and associative interference. Current Biology, 16(13), 1290-1294. doi: 10.1016/j.cub.2006.05.024.

Fell, J., \& Axmacher, N. (2011). The role of phase synchronization in memory processes. Nature Reviews Neuroscience, 12(2), 105-118. doi: 10.1038/nrn2979.

Fell, J., Staresina, B. P., Do Lam, A. T., Widman, G., Helmstaedter, C., Elger, C. E., \& Axmacher, N. (2013). Memory modulation by weak synchronous deep brain stimulation: A pilot study. Brain Stimulation, 6(3), 270-273. doi: 10.1016/ j.brs.2012.08.001.

Fletcher, P. C., \& Henson, R. N. A. (2001). Frontal lobes and human memory: Insights from functional neuroimaging. Brain, 124(5), 849-881. doi: 10.1093/brain/124.5.849.

Frase, L., Piosczyk, H., Zittel, S., Jahn, F., Selhausen, P., Krone, L., Feige, B., Mainberger, F., Maier, J. G., Kuhn, M., Klöppel, S., Normann, C., Sterr, A., Spiegelhalder, K., Riemann, D., Nitsche, M. A., \& Nissen, C. (2016). Modulation of total sleep time by transcranial direct current stimulation (tDCS). Neuropsychopharmacology, 41(10), 2577-2586. doi: 10.1038/npp.2016.65.

Fries, P. (2005). A mechanism for cognitive dynamics: Neuronal communication through neuronal coherence. Trends in Cognitive Sciences,9(10), 474-480. doi: 10.1016/j.tics.2005.08.011.

Gais, S., \& Born, J. (2004). Declarative memory consolidation: mechanisms acting during human sleep. Learning \& Memory, 11(6), 679-685. doi: 10.1101/1m.80504.

Gais, S., Plihal, W., Wagner, U., \& Born, J. (2000). Early sleep triggers memory for early visual discrimination skills. Nature Neuroscience,3(12), 1335-1339. doi: $10.1038 / 81881$.

Galli, G., Feurra, M., Pavone, E. F., Sirota, M., \& Rossi, S. (2017). Dynamic changes in prefrontal cortex involvement during verbal episodic memory formation. Biological Psychology, 125, 36-44. doi: 10.1016/j.biopsycho.2017.02.008. 
Gathercole, S. E., \& Baddeley, A. D. (2014). Working memory and language. New York: Psychology Press.

Giuditta, A., Ambrosini, M. V., Montagnese, P., Mandile, P., Cotugno, M., Zucconi, G. G., \& Vescia, S. (1995). The sequential hypothesis of the function of sleep. Behavioural Brain Research, 69(1-2), 157-166. doi: 10.1016/0166-4328(95)00012-I.

Godden, D. R., \& Baddeley, A. D. (1975). Context-dependent memory in two natural environments: On land and underwater. British Journal of Psychology, 66(3), 325331. doi: 10.1111/j.2044-8295.1975.tb01468.x.

Goldman-Rakic, P. S., Selemon, L. D., \& Schwartz, M. L. (1984). Dual pathways connecting the dorsolateral prefrontal cortex with the hippocampal formation and parahippocampal cortex in the rhesus monkey. Neuroscience, 12(3), 719-743. doi: 10.1016/0306-4522(84)90166-0.

Groch, S., Wilhelm, I., Diekelmann, S., \& Born, J. (2013). The role of REM sleep in the processing of emotional memories: Evidence from behavior and event-related potentials. Neurobiology of Learning and Memory, 99, 1-9. doi: 10.1016/j.nlm.2012.10.006.

Hanslmayr, S., \& Staudigl, T. (2014). How brain oscillations form memories - A processing based perspective on oscillatory subsequent memory effects. Neuroimage, 85, 648655. doi: 10.1016/j.neuroimage.2013.05.121.

Helfrich, R. F., Mander, B. A., Jagust, W. J., Knight, R. T., \& Walker, M. P. (2018). Old brains come uncoupled in sleep: Slow wave-spindle synchrony, brain atrophy, and forgetting. Neuron, 97(1), 221-230. doi: 10.1016/j.neuron.2017.11.020.

Helfrich, R. F., Schneider, T. R., Rach, S., Trautmann-Lengsfeld, S. A., Engel, A. K., \& Herrmann, C. S. (2014). Entrainment of brain oscillations by transcranial alternating current stimulation. Current Biology, 24(3), 333-339. doi: 10.1016/j.cub.2013.12.041.

Herwig, U., Satrapi, P., \& Schönfeldt-Lecuona, C. (2003). Using the international 10-20 EEG system for positioning of transcranial magnetic stimulation. Brain Topography, 16(2), 95-99. doi: 10.1023/B:BRAT.0000006333.93597.9d.

Hobson, J. A., \& Pace-Schott, E. F. (2002). The cognitive neuroscience of sleep: Neuronal systems, consciousness and learning. Nature Reviews Neuroscience, 3(9), 679-693. doi: 10.1038/nrn915. 
Hoddes, E., Zarcone, V., Smythe, H., Phillips, R., \& Dement, W. C. (1973). Quantification of sleepiness: A new approach. Psychophysiology, 10(4), 431-436. doi: 10.1111/j.14698986.1973.tb00801.x.

Homan, R. W., Herman, J., \& Purdy, P. (1987). Cerebral location of international 10-20 system electrode placement. Electroencephalography and Clinical Neurophysiology, 66(4), 376-382. doi: 10.1016/0013-4694(87)90206-9.

Jafarpour, A., Fuentemilla, L., Horner, A. J., Penny, W., \& Duzel, E. (2014). Replay of very early encoding representations during recollection. Journal of Neuroscience, 34(1), 242-248. doi: 10.1523/JNEUROSCI.1865-13.2014.

Javadi, A. H., \& Cheng, P. (2013). Transcranial direct current stimulation (tDCS) enhances reconsolidation of long-term memory. Brain Stimulation, 6(4), 668-674. doi: 10.1016/j.brs.2012.10.007.

Javadi, A. H., Glen, J. C., Halkiopoulos, S., Schulz, M., \& Spiers, H. J. (2017). Oscillatory reinstatement enhances declarative memory. Journal of Neuroscience, 37(41), 99399944. doi: 10.1523/JNEUROSCI.0265-17.2017.

Javadi, A. H., \& Walsh, V. (2012). Transcranial direct current stimulation (tDCS) of the left dorsolateral prefrontal cortex modulates declarative memory. Brain Stimulation, 5(3), 231-241. doi: 10.1016/j.brs.2011.06.007.

Jonides, J., Lewis, R. L., Nee, D. E., Lustig, C. A., Berman, M. G., \& Moore, K. S. (2008). The mind and brain of short-term memory. Annual Review of Psychology, 59, 193224. doi: 10.1146/annurev.psych.59.103006.093615.

Jutras, M. J., \& Buffalo, E. A. (2010). Synchronous neural activity and memory formation. Current Opinion in Neurobiology,20(2), 150-155. doi: 10.1016/j.conb.2010.02.006.

Kensinger, E. A., \& Corkin, S. (2003). Memory enhancement for emotional words: Are emotional words more vividly remembered than neutral words. Memory \& Cognition, 31(8), 1169-1180. doi: 10.3758/BF03195800.

Keppel, G., \& Underwood, B. J. (1962). Proactive inhibition in short-term retention of single items. Journal of Verbal Learning and Verbal Behavior, 1(3), 153-161. doi: 10.1016/S0022-5371(62)80023-1.

Ladenbauer, J., Ladenbauer, J., Külzow, N., de Boor, R., Avramova, E., Grittner, U., \& Flöel, A. (2017). Promoting sleep oscillations and their functional coupling by transcranial 
stimulation enhances memory consolidation in mild cognitive impairment. Journal of Neuroscience, 260-217. doi: 10.1523/JNEUROSCI.0260-17.2017.

Ladenbauer, J., Külzow, N., Paßmann, S., Antonenko, D., Grittner, U., Tamm, S., \& Flöel, A. (2016). Brain stimulation during an afternoon nap boosts slow oscillatory activity and memory consolidation in older adults. NeuroImage, 142, 311-323. doi: 10.1016/j.neuroimage.2016.06.057.

Lisman, J. (2010). Working memory: The importance of theta and gamma oscillations. Current Biology, 20(11), R490-R492. doi: 10.1016/j.cub.2010.04.011.

Manenti, R., Brambilla, M., Petesi, M., Ferrari, C., \& Cotelli, M. (2013). Enhancing verbal episodic memory in older and young subjects after non-invasive brain stimulation. Frontiers in Aging Neuroscience, 5(49), 1-9. doi: 10.3389/fnagi.2013.00049.

Maquet, P. (2001). The role of sleep in learning and memory. Science, 294(5544), 10481052. doi: 10.1126/science.1062856.

Marr, D. (1971). Simple memory: A theory for archicortex. Philosophical Transactions of the Royal Society B: Biological Sciences, 262(841), 23-81. doi: 10.1098/rstb.1971.0078.

Marshall, L., \& Born, J. (2007). The contribution of sleep to hippocampus-dependent memory consolidation. Trends in Cognitive Sciences, 11(10), 442-450. doi: 10.1016/ j.tics.2007.09.001.

Marshall, L., Helgadóttir, H., Mölle, M., \& Born, J. (2006). Boosting slow oscillations during sleep potentiates memory. Nature, 444(7119), 610-613. doi: 10.1038/nature05278.

Marshall, L., Kirov, R., Brade, J., Mölle, M., \& Born, J. (2011). Transcranial electrical currents to probe EEG brain rhythms and memory consolidation during sleep in humans. PloS one, 6(2), e16905. doi: 10.1371/journal.pone.0016905.

McClelland, J. L., McNaughton, B. L., \& O'Reilly, R. C. (1995). Why there are complementary learning systems in the hippocampus and neocortex: Insights from the successes and failures of connectionist models of learning and memory. Psychological Review, 102(3), 419-457. Retrieved from http://www.apa.org/pubs/journals/rev/.

Mednick, S., Nakayama, K., \& Stickgold, R. (2003). Sleep-dependent learning: A nap is as good as a night. Nature Neuroscience, 6(7), 697-698. doi: 10.1038/nn1078.

Medvedeva, A., Materassi, M., Neacsu, V., Beresford-Webb, J., Hussin, A., Khan, N., Newton, F., \& Galli, G. (2018). Effects of anodal transcranial direct current 
stimulation over the ventrolateral prefrontal cortex on episodic memory formation and retrieval. Cerebral Cortex, 1-9. doi: 10.1093/cercor/bhx347.

Mölle, M., Marshall, L., Gais, S., \& Born, J. (2002). Grouping of spindle activity during slow oscillations in human non-rapid eye movement sleep. Journal of Neuroscience, 22(24), 10941-10947. doi: 10.1523/JNEUROSCI.22-24-10941.2002.

Nadel, L., \& Moscovitch, M. (1997). Memory consolidation, retrograde amnesia and the hippocampal complex. Current Opinion in Neurobiology,7(2), 217-227. doi: 10.1016/S0959-4388(97)80010-4.

Nairne, J. S., Thompson, S. R., \& Pandeirada, J. N. (2007). Adaptive memory: Survival processing enhances retention. Journal of Experimental Psychology: Learning, Memory, and Cognition, 33(2), 263-271. doi: 10.1037/0278-7393.33.2.263.

Nitsche, M. A., Jakoubkova, M., Thirugnanasambandam, N., Schmalfuss, L., Hullemann, S., Sonka, K., Paulus, W., Trenkwalder, C., \& Happe, S. (2010). Contribution of the premotor cortex to consolidation of motor sequence learning in humans during sleep. Journal of Neurophysiology, 104(5), 2603-2614. doi: 10.1152/jn.00611.2010.

Norman, K. A., \& O'Reilly, R. C. (2003). Modeling hippocampal and neocortical contributions to recognition memory: A complementary-learning-systems approach. Psychological Review, 110(4), 611-646. doi: 10.1037/0033295X.110.4.611.

Nyhus, E., \& Badre, D. (2015). Functional organization of frontal cortex. In D. R. Addis, M. Barense, \& A. Duarte (Eds.), The Wiley handbook on the cognitive neuroscience of memory (pp. 131-149). West Sussex, UK: John Wiley \& Sons.

Nyhus, E., \& Curran, T. (2010). Functional role of gamma and theta oscillations in episodic memory. Neuroscience \& Biobehavioral Reviews,34(7), 1023-1035. doi: 10.1016/j.neubiorev.2009.12.014.

O’Neill, J., Pleydell-Bouverie, B., Dupret, D., \& Csicsvari, J. (2010). Play it again: Reactivation of waking experience and memory. Trends in Neurosciences, 33(5), 220229. doi: 10.1016/j.tins.2010.01.006.

O'Reilly, R. C., \& Rudy, J. W. (2001). Conjunctive representations in learning and memory: Principles of cortical and hippocampal function. Psychological Review, 108(2), 311345. doi: .10.1037/0033-295X.108.2.311.

Osipova, D., Takashima, A., Oostenveld, R., Fernández, G., Maris, E., \& Jensen, O. (2006). Theta and gamma oscillations predict encoding and retrieval of declarative 
memory. Journal of Neuroscience, 26(28), 7523-7531. doi: 10.1523/JNEUROSCI. 1948-06.2006.

Oudiette, D., \& Paller, K. A. (2013). Upgrading the sleeping brain with targeted memory reactivation. Trends in Cognitive Sciences, 17(3), 142-149. doi: 10.1016/j.tics.2013.01.006.

Paßmann, S., Külzow, N., Ladenbauer, J., Antonenko, D., Grittner, U., Tamm, S., \& Flöel, A. (2016). Boosting slow oscillatory activity using tDCS during early nocturnal slow wave sleep does not improve memory consolidation in healthy adults. Brain Stimulation, 9, 730-739. doi: 10.1016/j.brs.2016.04.016.

Peigneux, P., Laureys, S., Delbeuck, X., \& Maquet, P. (2001). Sleeping brain, learning brain. The role of sleep for memory systems. Neuroreport, 12(18), A111-A124. doi: 10.1097/00001756-200112210-00001.

Peigneux, P., Laureys, S., Fuchs, S., Collette, F., Perrin, F., Reggers, J., Phillips, C., Degueldre, C., Del Fiore, G., Aerts, J., Luxen, A., \& Maquet, P. (2004). Are spatial memories strengthened in the human hippocampus during slow wave sleep?. Neuron, 44(3), 535-545. doi: 10.1016/j.neuron.2004.10.007.

Petersen, R. C., Caracciolo, B., Brayne, C., Gauthier, S., Jelic, V., \& Fratiglioni, L. (2014). Mild cognitive impairment: A concept in evolution. Journal of Internal Medicine, 275(3), 214-228. doi: 10.1111/joim.12190.

Peterson, L., \& Peterson, M. J. (1959). Short-term retention of individual verbal items. Journal of Experimental Psychology, 58(3), 193-198. doi: 10.1037/h0049234.

Petrides, M., \& Pandya, D. N. (1999). Dorsolateral prefrontal cortex: Comparative cytoarchitectonic analysis in the human and the macaque brain and corticocortical connection patterns. European Journal of Neuroscience, 11(3), 1011-1036. doi: 10.1046/j.1460-9568.1999.00518.x.

Plihal, W., \& Born, J. (1997). Effects of early and late nocturnal sleep on declarative and procedural memory. Journal of Cognitive Neuroscience,9(4), 534-547. doi: 10.1162/jocn.1997.9.4.534.

Plihal, W., \& Born, J. (1999). Effects of early and late nocturnal sleep on priming and spatial memory. Psychophysiology, 36(5), 571-582. doi: 10.1111/1469-8986.3650571.

Rasch, B., \& Born, J. (2013). About sleep's role in memory. Physiological Reviews, 93(2), 681-766. doi: 10.1152/physrev.00032.2012. 
Rasch, B., Büchel, C., Gais, S., \& Born, J. (2007). Odor cues during slow-wave sleep prompt declarative memory consolidation. Science, 315(5817), 1426-1429. doi: 10.1126/science. 113858 .

Rauchs, G., Bertran, F., Guillery-Girard, B., Desgranges, B., Kerrouche, N., Denise, P., Foret, J., \& Eustache, F. (2004). Consolidation of strictly episodic memories mainly requires rapid eye movement sleep. Sleep, 27(3), 395-401. doi: 10.1093/sleep/27.3.395.

Rauchs, G., Schabus, M., Parapatics, S., Bertran, F., Clochon, P., Hot, P., Denise, P., Desgranges, B., Eustache, F., Gruber, G., \& Anderer, P. (2008). Is there a link between sleep changes and memory in Alzheimer's disease?. Neuroreport, 19(11), 1159-1162. doi: 10.1097/WNR.0b013e3283 0867c4.

Ray, R. D., \& Zald, D. H. (2012). Anatomical insights into the interaction of emotion and cognition in the prefrontal cortex. Neuroscience \& Biobehavioral Reviews, 36(1), 479-501. doi: 10.1016/j.neubiorev.2011.08.005.

Rechtschaffen, A., \& Kales, A. (1968). A Manual of Standardized Terminology, Techniques and Scoring System for Sleep Stages of Human Subjects. Bethesda, Maryland: US National Institute of Neurological Diseases and Blindness, Neurological Information Network.

Ricker, T. J., \& Cowan, N. (2010). Loss of visual working memory within seconds: The combined use of refreshable and non-refreshable features. Journal of Experimental Psychology: Learning, Memory, and Cognition,36(6), 1355-1368. doi: $10.1037 / \mathrm{a} 0020356$.

Rivera-Urbina, G. N., Nitsche, M. A., \& Molero-Chamizo, A. (2016). Transcranial direct current stimulation (tDCS) in the context of sleep and insomnia. Dreaming, 3(5), 1060-1065. Retrieved from http://www.apa.org/pubs/journals/drm/.

Roark, B., Mitchell, M., Hosom, J-P., Hollingshead, K., \& Kaye, J. (2011). Spoken language derived measures for detecting mild cognitive impairment. IEEE Transactions on Audio, Speech, and Language Processing, 19(7), 2081-2090. doi: 10.1109/TASL.2011.2112351.

Schott, B. H., Niklas, C., Kaufmann, J., Bodammer, N. C., Machts, J., Schütze, H., \& Düzel, E. (2011). Fiber density between rhinal cortex and activated ventrolateral prefrontal regions predicts episodic memory performance in humans. Proceedings of the National Academy of Sciences, 108(13), 5408-5413. doi: 10.1073/pnas.1013287108. 
Scullin, M. K. (2013). Sleep, memory, and aging: the link between slow-wave sleep and episodic memory changes from younger to older adults. Psychology and Aging, 28(1), 105-114. doi: 10.1037/a0028830.

Sederberg, P. B., Kahana, M. J., Howard, M. W., Donner, E. J., \& Madsen, J. R. (2003). Theta and gamma oscillations during encoding predict subsequent recall. Journal of Neuroscience, 23(34), 10809-10814. doi: 10.1523/JNEUROSCI.23-34-10809.2003.

Sederberg, P. B., Schulze-Bonhage, A., Madsen, J. R., Bromfield, E. B., Litt, B., Brandt, A., \& Kahana, M. J. (2007). Gamma oscillations distinguish true from false memories. Psychological Science, 18(11), 927-932. doi: 10.1111/j.14679280.2007.02003.x.

Shimizu, R. E., Connolly, P. M., Cellini, N., Armstrong, D. M., Hernandez, L. T., Estrada, R., Aguilar, M., Weisend, M. P., Mednick, S. C., \& Simons, S. B. (2018). Closedloop targeted memory reactivation during sleep improves spatial navigation. Frontiers in Human Neuroscience, 12(28), 1-14. doi: 10.3389/fn-hum.2018.00028.

Siegel, M., Donner, T. H., \& Engel, A. K. (2012). Spectral fingerprints of large-scale neuronal interactions. Nature Reviews Neuroscience, 13(2), 121-134. doi: 10.1038/nrn3137.

Smith, S. M., \& Vela, E. (2001). Environmental context-dependent memory: A review and meta-analysis. Psychonomic Bulletin \& Review, 8(2), 203-220. doi: 10.3758/BF03196157.

Smith, C., \& Weeden, K. (1990). Post training REMs coincident auditory stimulation enhances memory in humans. Psychiatric Journal of the University of Ottawa, 15(2), 85-90. Retrieved from https://www.ncbi.nlm.nih.gov/ labs/journals/psychiatr-j-univott/.

Squire, L. R. (1992). Declarative and nondeclarative memory: Multiple brain systems supporting learning and memory. Journal of Cognitive Neuroscience, 4(3), 232-243. doi: 10.1162/jocn.1992.4.3.232.

Squire, L. R., \& Zola, S. M. (1996). Structure and function of declarative and nondeclarative memory systems. Proceedings of the National Academy of Sciences, 93(24), 1351513522. doi: 10.1073/pnas.93.24.13515.

Staresina, B. P., Bergmann, T. O., Bonnefond, M., Van Der Meij, R., Jensen, O., Deuker, L., Elger, C. E., Axmacher, N., \& Fell, J. (2015). Hierarchical nesting of slow 
oscillations, spindles and ripples in the human hippocampus during sleep. Nature Neuroscience, 18(11), 1679-1686. doi: 10.1038/nn.4119.

Staresina, B. P., Michelmann, S., Bonnefond, M., Jensen, O., Axmacher, N., \& Fell, J. (2016). Hippocampal pattern completion is linked to gamma power increases and alpha power decreases during recollection. Elife, 5,1-18. doi: 10.7554/eLife.17397.

Staudigl, T., \& Hanslmayr, S. (2018). Reactivation of neural patterns during memory reinstatement supports encoding specificity. bioRxiv. doi: 10.1101/255166.

Strüber, D., Rach, S., Trautmann-Lengsfeld, S. A., Engel, A. K., \& Herrmann, C. S. (2014). Antiphasic $40 \mathrm{~Hz}$ oscillatory current stimulation affects bistable motion perception. Brain Topography, 27(1), 158-171. doi: 10.1007/s10548-013-0294-x.

Sutterer, D. W., Foster, J. J., Serences, J. T., Vogel, E. K., \& Awh, E. (2018). Alpha-band oscillations track the retrieval of precise spatial representations from long-term memory. bioRxiv. doi: 10.1101/207860.

Tamminen, J., Ralph, M. A. L., \& Lewis, P. A. (2017). Targeted memory reactivation of newly learned words during sleep triggers REM-mediated integration of new memories and existing knowledge. Neurobiology of Learning and Memory, 137, 7782. doi: 10.1016/j.nlm.2016.11.012.

Teyler, T. J., \& DiScenna, P. (1985). The role of hippocampus in memory: A hypothesis. Neuroscience \& Biobehavioral Reviews, 9(3), 377-389. doi: 10.1016/01497634(85)90016-8.

Teyler, T. J., \& Rudy, J. W. (2007). The hippocampal indexing theory and episodic memory: Updating the index. Hippocampus, 17(12), 1158-1169. doi: 10.1002/hipo.20350.

Tulving, E., \& Thomson, D. M. (1973). Encoding specificity and retrieval processes in episodic memory. Psychological Review, 80(5), 352-373. doi: 10.1037/h0020071.

von Stein, A., \& Sarnthein, J. (2000). Different frequencies for different scales of cortical integration: From local gamma to long range alpha/theta synchronization. International Journal of Psychophysiology, 38(3), 301-313. doi: 10.1016/S0167-8760(00)00172-0.

Wagner, U., Hallschmid, M., Verleger, R., \& Born, J. (2003). Signs of REM sleep dependent enhancement of implicit face memory: a repetition priming study. Biological Psychology, 62(3), 197-210. doi: 10.1016/S0301-0511(02)00125-4.

Walker, M. P., \& Stickgold, R. (2006). Sleep, memory, and plasticity. Annual Review of Psychology, 57, 139-166. doi: 1146/annurev.psych.56.091103.070307. 
Wang, H. C., Savage, G., Gaskell, M. G., Paulin, T., Robidoux, S., \& Castles, A. (2017). Bedding down new words: Sleep promotes the emergence of lexical competition in visual word recognition. Psychonomic Bulletin \& Review, 24(4), 1186-1193. doi: 10.3758/s13423-016-1182-7.

Watrous, A. J., \& Ekstrom, A. D. (2014). The spectro-contextual encoding and retrieval theory of episodic memory. Frontiers in Human Neuroscience, 8(75), 1-15. doi: 10.3389/fnhum.2014.00075.

Watrous, A. J., Fell, J., Ekstrom, A. D., \& Axmacher, N. (2015). More than spikes: Common oscillatory mechanisms for content specific neural representations during perception and memory. Current Opinion in Neurobiology,31, 33-39. doi: 10.1016/j.conb.2014.07.024.

Watrous, A. J., Miller, J., Qasim, S. E., Fried, I., \& Jacobs, J. (2018). Phase-tuned neuronal firing encodes human contextual representations for navigational goals. eLife, 7 , e32554. doi: 10.7554/eLife.32554.

Watrous, A. J., Tandon, N., Conner, C. R., Pieters, T., \& Ekstrom, A. D. (2013). Frequencyspecific network connectivity increases underlie accurate spatiotemporal memory retrieval. Nature Neuroscience, 16(3), 349-356. doi: 10.1038/nn.3315.

Westerberg, C. E., Mander, B. A., Florczak, S. M., Weintraub, S., Mesulam, M. M., Zee, P. C., \& Paller, K. A. (2012). Concurrent impairments in sleep and memory in amnestic mild cognitive impairment. Journal of the International Neuropsychological Society, 18(3), 490-500. doi: 10.1017/S135561771200001X.

Wimber, M., Maaß, A., Staudigl, T., Richardson-Klavehn, A., \& Hanslmayr, S. (2012). Rapid memory reactivation revealed by oscillatory entrainment. Current Biology, 22(16), 1482-1486. doi: 10.1016/j.cub.2012.05.054.

Womelsdorf, T., Schoffelen, J. M., Oostenveld, R., Singer, W., Desimone, R., Engel, A. K., $\&$ Fries, P. (2007). Modulation of neuronal interactions through neuronal synchronization. Science, 316(5831), 1609-1612. doi: 10.1126/science.1139597. 